\title{
Analysis of Epidemiological Indices of Coronavirus Disease 2019 (COVID-19) in Countries with High- incidence
}

Javad Javan-Noughabi

Mashhad University of Medical Sciences

Sayed Ali Mousavi

Shoushtar Faculty of Medical science

Ahmad Faramarzi (D Ahmadfaramarzi97@yahoo.com)

Urmia University of Medical Sciences

Hamidreza Shabanikiya

Mashhad University of Medical Sciences

Research article

Keywords: COVID-19, Incidence, Mortality, Fatality

Posted Date: November 18th, 2020

DOI: https://doi.org/10.21203/rs.3.rs-108683/v1

License: (a) (i) This work is licensed under a Creative Commons Attribution 4.0 International License.

Read Full License 


\section{Abstract}

Background: Coronavirus disease 2019 (COVID-19) poses an extraordinary threat to global public health. According to a report by WHO, COVID-19 has affected approximately 20 million patients in the world and has caused nearly 728 thousand deaths as of 10 August 2020. The purpose of the current study was to provide an estimate and comparison of epidemiological indices on COVID-19 in 10 countries with highincidence.

Methods: We did a descriptive and comparative study intending to analyze the epidemiological indices of COVID-19 in countries with high-incidence, using the data published by the WHO until August 10, 2020. We estimated the incidence and mortality rate per 100,000 population on a daily and general level.

Results: The mean of fatality rate in 10 countries was almost $3.26 \%$. The highest value on the fatality rate was obtained with $10.93 \%$ in Mexico, followed by $5.64 \%$ in Iran and 4.43 in Peru. The lowest value on the fatality rate is related to the Russian Federation and South Africa at $1.68 \%$ and $1.86 \%$, respectively. Chile had the highest incidence rate with 1951 cases per 100,000 population. This rate is followed by 1496, 1428 and 1417 in the United States of America (USA), Peru and Brazil, respectively.

Conclusions: Until August 10, 2020, COVID-19 has affected 13.7 million patients and caused 445.2 thousand deaths in 10 countries with high-incidence. This study shows that each country has a specific pattern of COVID-19.

\section{Introduction}

The ongoing epidemic of the new coronavirus, coronavirus disease 2019 (COVID-19), poses an extraordinary threat to global public health (1). During the past two decades, the world has experienced two major epidemics from two different beta coronaviruses; severe acute respiratory syndrome coronavirus (SARS-CoV) and Middle East respiratory syndrome coronavirus (MERS-CoV). In total, these two outbreaks have resulted in more than 10,000 cumulative cases, with fatality rates of $10 \%$ for SARSCoV and $37 \%$ for MERS-CoV (2).

The COVID-19, identified in China at the end of 2019, has a high contagion potential, and its incidence has increased exponentially. Its widespread transmission was recognized by the World Health Organization (WHO) as a pandemic (3). According to a report by WHO, COVID-19 has affected approximately 20 million patients in the world and has caused nearly 728 thousand deaths as of 10 August 2020 (4). In addition to the health consequences of COVID-19, it will change the world's economy and this change will be the source of creating distance between countries depending on their approaches in dealing with disease, the type of interventions, diagnostic methods and control tools (5).

More data are required to illustrate the full spectrum of the epidemiological characteristics of the outbreak (6). On the other hand, there have been many studies on COVID-19 epidemiology in the world, most of these studies have been conducted in a country or at a specific regional (7-9). Therefore, the 
present study aims to provide an estimate and comparison of epidemiological indices on COVID-19 in 10 countries with high-incidence.

\section{Methods}

We did a descriptive and comparative study intending to analyze the epidemiological indices of COVID-19 in countries with high-incidence. For this purpose, we selected 10 countries that had the highest incidence cases on August 10. In other words, they had the highest cumulative number of COVID-19 until 10 August, 2020. The list of countries includes the United States of America (USA), Brazil, India, the Russian Federation, South Africa, Mexico, Peru, Colombia, Chile and Iran. The research data were collected from the World Health Organization (WHO) website (4). The WHO publishes the reports on COVID-19 based on the number of diagnosed and death cases in all counties. The date of the first reported case on COVID-19 in the USA, India and the Russian Federation was on January 20th, 30th and 31th, respectively. The first case was observed in Iran, Brazil and Mexico on February 19th, 26th and 28th, respectively. In Chile, South Africa, Colombia and Peru, the first reported on COVID-19 was in March, on the 3rd, 5th, 6th and 7th, respectively.

We examined the COVID-19 trend in the selected countries in two stages. In the first stage, we extracted the daily number of confirmed incidence cases and the death number for selected countries until 10 August. Then, we calculated the cumulative cases on incidence and death for COVID-19. We estimated the incidence and mortality rate per 100,000 population on a daily and general level. For this purpose, we applied the population of listed countries in 2020 using the United Nations reports (10). The following formulas were used for incidence and mortality rates.

\section{A: Incidence rate $=\frac{\text { The number of confirmed cases }}{\text { The population in } 2020} \times 100,000$}

\section{B: Mortality rate $=\frac{\text { The number of death due to COVID }-19}{\text { The population in } 2020} \times 100,000$}

We also extracted the fatality rate at this stage by dividing the number of deaths from COVID-19 disease by the total number of infected people.

In the second stage, we estimated the average weekly incidence and mortality rates on COVID-19 for the samples studied. We then depicted trends at these rates in each country. We took the weekly average to compare trends for the following reasons. First, diagnostic test results by COVID-19 are usually reported with a delay, especially in the early of an epidemic. The needed equipment to diagnose the disease at the onset of epidemics is usually not available or is rarely available in the capital of countries. Daily statistics are likely to have errors; therefore we used the weekly mean to solve this problem. Second, the incubation period by COVID-19 is between 5 to 14-day, which most studies have reported as 7-day (11-13). 
Tables and graphs were used to analyze the trend of epidemiological indicators. All the analyses were conducted using Stata 14 (Stata Corp, College Station, Tex) software.

\section{Results}

Table 1 presents the total case, total death, and fatality rate by COVID-19 in 10 countries with highincidence until August 10, 2020. The USA had the highest total cases of COVID-19 (about 5 million), and the Islamic Republic of Iran had the lowest (around 327 thousand) at the follow-up time. The highest and lowest total death due to COVID-19 was related to the USA and Chile respectively, but the highest and lowest fatality rate was in Mexico (10.93\%) and the Russian Federation (1.68\%), respectively.

Table 1

The number of incidence and death of COVID-19 by 10 August, 2020 in selected countries

\begin{tabular}{|llll|}
\hline Countries & Total case reported & Total death reported & $\begin{array}{l}\text { Fatality rate } \\
\text { (\%) }\end{array}$ \\
\hline United states & $4,951,851$ & 160,989 & 3.25 \\
\hline Brazil & $3,012,412$ & 100,477 & 3.34 \\
\hline India & $2,215,074$ & 44,386 & 2.00 \\
\hline Russian Federation & 892,654 & 15,001 & 1.68 \\
\hline South Africa & 559,859 & 10,408 & 1.86 \\
\hline Mexico & 475,902 & 52,006 & 10.93 \\
\hline Peru & 471,012 & 20,844 & 4.43 \\
\hline Colombia & 376,870 & 12,540 & 3.33 \\
\hline Chile & 373,056 & 10,077 & 2.70 \\
\hline Iran & 326,712 & 18,427 & 5.64 \\
\hline Total & $13,655,402$ & 445,155 & 3.26 \\
\hline
\end{tabular}

Table 2 shows the daily average for incidence and mortality rate per 100,000 population until 10 August 2020, in selected countries. According to Table 2, the daily incidence of COVID-19 cases ranged from 0.83 per 100,000 population for India to 12.12 per 100,000 population for Chile. The highest and lowest daily mortality rate on COVID-19 was related to Peru (0.4 per 100,000 population) and India (0.017 per 100,000 population) respectively. 
Table 2

The average daily incidence and mortality rate per 100,000 population until 10 August, 2020 in selected countries

\begin{tabular}{|c|c|c|c|c|c|c|}
\hline \multirow[t]{2}{*}{ Countries } & \multicolumn{3}{|l|}{ Incidence } & \multicolumn{3}{|l|}{ Mortality } \\
\hline & Mean & Maximum & Total & Mean & Maximum & Total \\
\hline \multirow{2}{*}{$\begin{array}{l}\text { United } \\
\text { states }\end{array}$} & 7.33 & 22.47 & 1496.02 & 0.24 & 1.94 & 43.64 \\
\hline & $(6.43-8.23)$ & & & $(02-0.27)$ & & \\
\hline \multirow[t]{2}{*}{ Brazil } & 8.49 & 32.50 & 1417.21 & 0.28 & 0.75 & 47.27 \\
\hline & $(7.17-9.81)$ & & & $(0.25-0.32)$ & & \\
\hline \multirow[t]{2}{*}{ India } & 0.83 & 4.67 & 160.51 & 0.017 & 0.15 & 3.22 \\
\hline & $(0.65-1.00)$ & & & $(0.01-0.02)$ & & \\
\hline \multirow{2}{*}{$\begin{array}{l}\text { Russian } \\
\text { Federation }\end{array}$} & 3.17 & 8.61 & 611.68 & 0.05 & 0.16 & 10.28 \\
\hline & $(2.79-3.55)$ & & & $(0.04-0.06)$ & & \\
\hline \multirow{2}{*}{$\begin{array}{l}\text { South } \\
\text { Africa }\end{array}$} & 5.94 & 23.51 & 943.97 & 0.11 & 0.96 & 17.55 \\
\hline & $(4.78-7.09)$ & & & $(0.08-0.14)$ & & \\
\hline \multirow[t]{2}{*}{ Mexico } & 2.24 & 7.41 & 369.11 & 0.24 & 0.85 & 40.34 \\
\hline & $(1.93-2.55)$ & & & $(0.21-0.28)$ & & \\
\hline \multirow[t]{2}{*}{ Peru } & 9.1 & 44.56 & 1428.53 & 0.4 & 11.75 & 63.22 \\
\hline & $(7.98-10.22)$ & & & $(0.25-0.55)$ & & \\
\hline \multirow[t]{2}{*}{ Colombia } & 4.69 & 23.58 & 740.66 & 0.16 & 0.75 & 24.64 \\
\hline & $(3.72-5.65)$ & & & $(0.12-0.19)$ & & \\
\hline \multirow[t]{2}{*}{ Chile } & 12.12 & 189.26 & 1951.52 & 0.33 & 5.53 & 52.71 \\
\hline & $(9.41-14.83)$ & & & $(0.24-0.42)$ & & \\
\hline \multirow[t]{2}{*}{ Iran } & 2.24 & 4.26 & 388.98 & 0.13 & 0.28 & 21.94 \\
\hline & $(2.09-2.38)$ & & & $(0.12-0.14)$ & & \\
\hline
\end{tabular}

The maximum of daily incidence and mortality rate was reported in Chile with 189 and Peru with 11.75 per 100,000 population, respectively. The highest total incidence rate per 100,000 population reported in Chile, followed by the USA, Peru and Brazil. The highest total mortality rate per 100,000 population reported in Peru followed by Chile, Brazil, the USA and Mexico.

In Fig. 1, we show the number of weekly incidence and death due to COVID-19. The weekly incidence in the majority of countries showed a significant ascending trend. However, the number of weekly incidence in the Russian Federation and Chile had a significant descending trend from the 16th week. Also, there 
was a decline in the trend of weekly incidence in South Africa from the 21st week. Although the USA, Peru and the Islamic Republic of Iran could decrease the weekly incidence of COVID-19 in some weeks, after that, we observed a rising trend in the number of weekly incidences of COVID-19.

The highest weekly incidence of COVID-19 occurred in the USA with more than 66,000 cases in a week (27th week). Besides, the highest number of weekly deaths reported in the USA with about 2600 cases in a week (13th week).

\section{Discussion}

In this paper, we provided an analysis of incidence and mortality indicators on COVID-19 in countries with high-incidence. The number of total cases was reported by approximately 13.7 million and there were 445.2 thousand deaths due to COVID-19 until 10 August, 2020. This report shows that the average fatality rate of COVID-19 in 10 countries with high-incidence is almost 3.26\%. Until August 10, 2020, about 20 million cases of COVID-19 with a total of 728 thousand deaths have been reported worldwide. This study indicates that these 10 countries were responsible for about $67 \%$ of COVID-19 cases and $60 \%$ of deaths.

Given the fatality rate in 10 countries with high-incidence, the highest value was obtained with $10.93 \%$ in Mexico, followed by $5.64 \%$ in Iran and 4.43 in Peru. Our study showed that until 10 August, Chile had the highest incidence rate with 1951 cases per 100,000 population. This rate is followed by 1496, 1428 and 1417 in the USA, Peru and Brazil, respectively. A comparison of mortality rate on COVID-19 in selected countries reveals that the highest mortality rate has existed in Peru with 63.22 deaths per 100,000 population. Then Chile, Brazil and the USA have the highest values with 52.71, 47.27 and 43.64. However, the analysis of daily data proves that Chile has the highest average daily on the incidence rate with 12.12 , and Peru with 0.4 death per 100,00 population has the first rank on daily mortality rate. The second and third rank in terms of the daily incidence rate is related to Peru and Brazil, followed by the USA. Although India with 160 cases per 100,000 population for incidence rate and 3.22 death for mortality rate had the lowest value, it does not have the last rank in terms of fatality rate. The lowest value on the fatality rate is related to the Russian Federation and South Africa at $1.68 \%$ and $1.86 \%$, respectively.

The fatality rate in COVID-19 is significantly lower than in SARS and MERS. Our analysis of the fatality rate in selected countries specified that the fatality rate is about $3.26 \%$ on average. While studies have reported a fatality rate of $9.6 \%$ and $36 \%$ for SARS and MERS (14-16). However, our study overestimated the fatality rate on COVID-19 for some countries. For example, it was estimated at $10.93 \%$ for Mexico and $5.64 \%$ in Iran. In these countries, all cases on COVID-19 have probably not been diagnosed, especially in countries where diagnostic testing technology was not available at the beginning of outbreak. Also, the incubation period in the COVID-19 is relatively long, so many cases at this disease do not show symptoms and go undiagnosed. Besides, a part of this variation on the fatality rate may be due to differences in population structure. 
Based on the results, distinct patterns emerged for the trend of incidence cases in selected countries. The first group is countries whose trends on the weekly mean of incidence number are still increasing and has not reached its maximum. These countries are India, Colombia, Brazil and Mexico. Another group such as the Russian Federation, South Africa and Chile, at first touched a peak on the incidence number, and then this trend declined steadily until August 10. Some countries, such as the USA, Iran, and Peru, have a unique pattern. In these countries, initially the incidence number has increased with a steep slope, but it has become decreasing and this cycle has been repeated. This result indicated that the trend of COVID-19 in these countries can be a function of different variables, including decisions that policymakers make to control this disease, the geographical location and economic status in countries, the availability of the health system to respond to the disease and cultural characteristics. For instance, the rapid growth in incidence cases during the 5th to 7th week in Iran could be due to travel caused by the New Year festival holidays.

In this study, we had some limitations. One major limitation of this study relates to the reported data by the WHO. These data couldn't reflect the actual incidence cases due to a large number of hidden cases in COVID-19. Therefore, it is likely that our study underestimated the incidence rate in selected countries. Also, the fatality rate is likely to be overestimated, especially in Mexico and Iran. Because in these countries the actual cases of COVID-19 should probably be much higher than the reported cases. Another shortcoming of this study relates to the onset timing at the COVID-19 outbreak in chosen countries. The date of epidemy onset on COVID-19 varies in the selected countries. The comparison of disease trends at different dates could be associated with distorted results.

\section{Conclusion}

Despite the mentioned limitation, to the best of our knowledge, this is the first research to compare epidemiological indices of COVID-19 in countries with high-incidence. Until August 10, 2020, COVID-19 has affected 13.7 million patients and caused 445.2 thousand deaths in 10 countries with high-incidence. This study shows that each country has a specific pattern of COVID-19 and these trends can be a function of different variables.

\section{Declarations}

\section{Acknowledgment}

All authors are appreciating from Mashhad University of Medical Sciences and we thank the World Health Organization team for providing data on COVID-19.

\section{Authors' contributions}

AF and JJN were the principal investigators of the research and designed the study. AF and SAM collected data, managed and analyzed the data. AF and HS drafted the first version of the manuscript, 
and all authors contributed, reviewed, and approved this paper.

\section{Funding}

This work was supported by the Mashhad University of Medical Sciences.

\section{Availability of data and materials}

The preliminary data on COVID-19 are available in the World Health Organization.

\section{Ethics approval and consent to participate}

This paper is the result of a research approved by Mashhad University of Medical Sciences.

\section{Consent for publication}

Not applicable.

\section{Conflict of Interests}

The authors declare that they have no competing interests.

\section{References}

1. Jamrozik E, Selgelid MJ. COVID-19 human challenge studies: ethical issues. The Lancet Infectious Diseases. 2020.

2. Kandel N, Chungong S, Omaar A, Xing J. Health security capacities in the context of COVID-19 outbreak: an analysis of International Health Regulations annual report data from 182 countries. The Lancet. 2020.

3. Ornell F, Schuch JB, Sordi AO, Kessler FHP. "Pandemic fear" and COVID-19: mental health burden and strategies. Brazilian Journal of Psychiatry. 2020;42(3):232-5.

4. WHO. Coronavirus Disease (COVID-19) Dashboard. 2020. Available from: https://covid19.who.int/table.

5. Baldwin R, Weder di Mauro B. Economics in the Time of COVID-19. CEPR Press; 2020.

6. Pan A, Liu L, Wang C, Guo H, Hao X, Wang Q, et al. Association of public health interventions with the epidemiology of the COVID-19 outbreak in Wuhan, China. Jama. 2020;323(19):1915-23.

7. Zhai P, Ding Y, Wu X, Long J, Zhong Y, Li Y. The epidemiology, diagnosis and treatment of COVID-19. International journal of antimicrobial agents. 2020:105955. 
8. Huang X, Wei F, Hu L, Wen L, Chen K. Epidemiology and clinical characteristics of COVID-19. Archives of Iranian medicine. 2020;23(4):268-71.

9. Roosa K, Lee Y, Luo R, Kirpich A, Rothenberg R, Hyman J, et al. Real-time forecasts of the COVID-19 epidemic in China from February 5th to February 24th, 2020. Infectious Disease Modelling. 2020;5:256-63.

10. United Nations. World Population Prospects 2019. 2020. Available from: http://creativecommons.org/licenses/by/3.0/igo/. .

11. Patrikar S, Kotwal A, Bhatti V, Banerjee A, Chatterjee K, Kunte R, et al. Incubation Period and Reproduction Number for novel coronavirus (COVID-19) infections in India. medRxiv. 2020.

12. Qin J, You C, Lin Q, Hu T, Yu S, Zhou X-H. Estimation of incubation period distribution of COVID-19 using disease onset forward time: a novel cross-sectional and forward follow-up study. medRxiv. 2020.

13. McAloon C, Collins Á, Hunt K, Barber A, Byrne AW, Butler F, et al. Incubation period of COVID-19: a rapid systematic review and meta-analysis of observational research. BMJ open. 2020;10(8):e039652.

14. Shahrajabian MH, Sun W, Cheng Q. Product of natural evolution (SARS, MERS, and SARS-CoV-2); deadly diseases, from SARS to SARS-CoV-2. Human Vaccines \& Immunotherapeutics. 2020:1-22.

15. WHO. Update 49 - SARS case fatality ratio, incubation period. 2003. Available from: https://www.who.int/csr/sars/ archive/2003_05_07a/en/.

16. Donnelly C, Malik M, Elkholy A, Cauchemez S, Van Kerkhove M. Important reductions in the global number of MERS cases and deaths since 2016. Emerging Infectious Diseases. 2019;25(9).

\section{Figures}




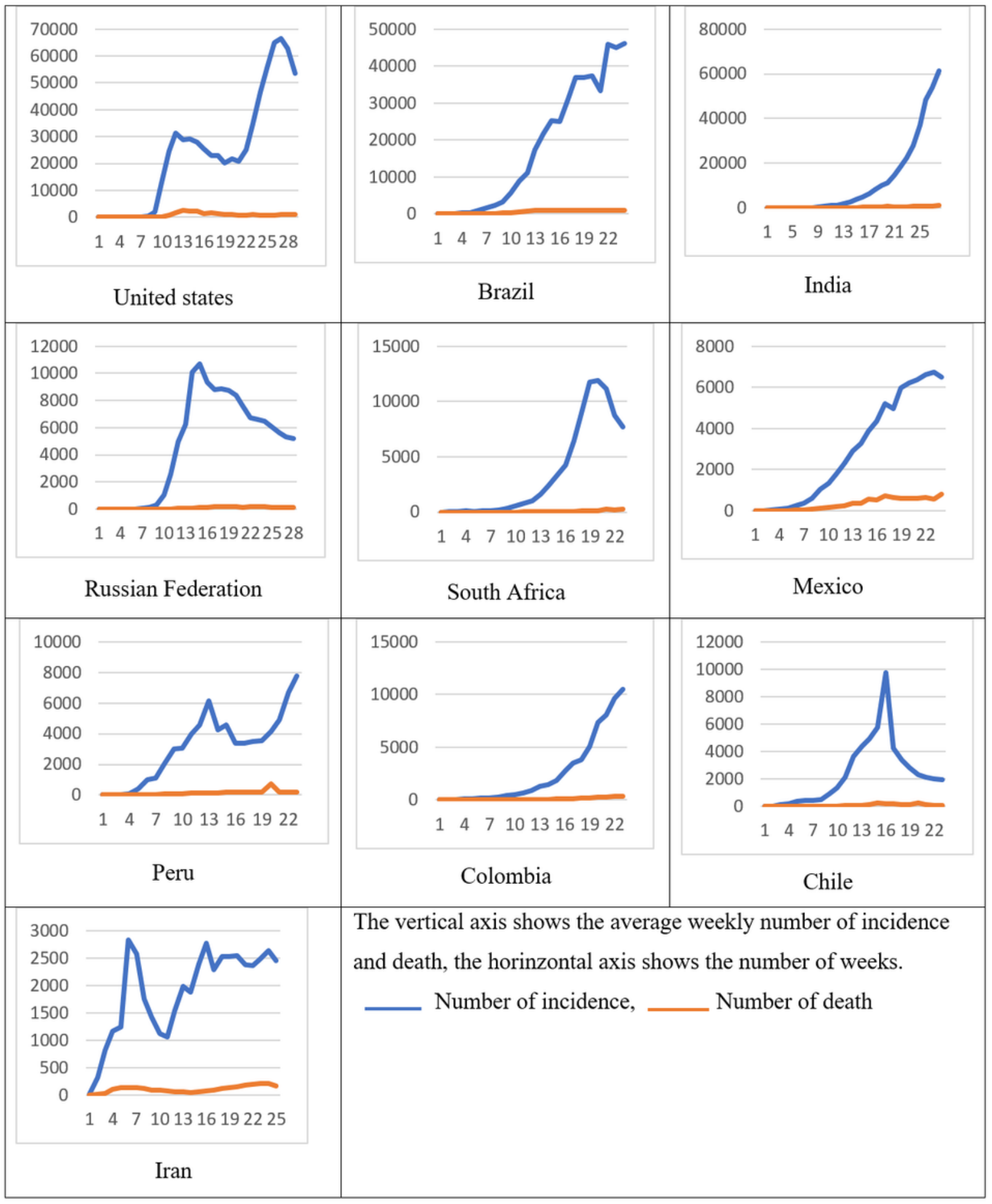

\section{Figure 1}

The trend in the number of incidence and death due to COVID-19 until 10 August, 2020 in selected countries 


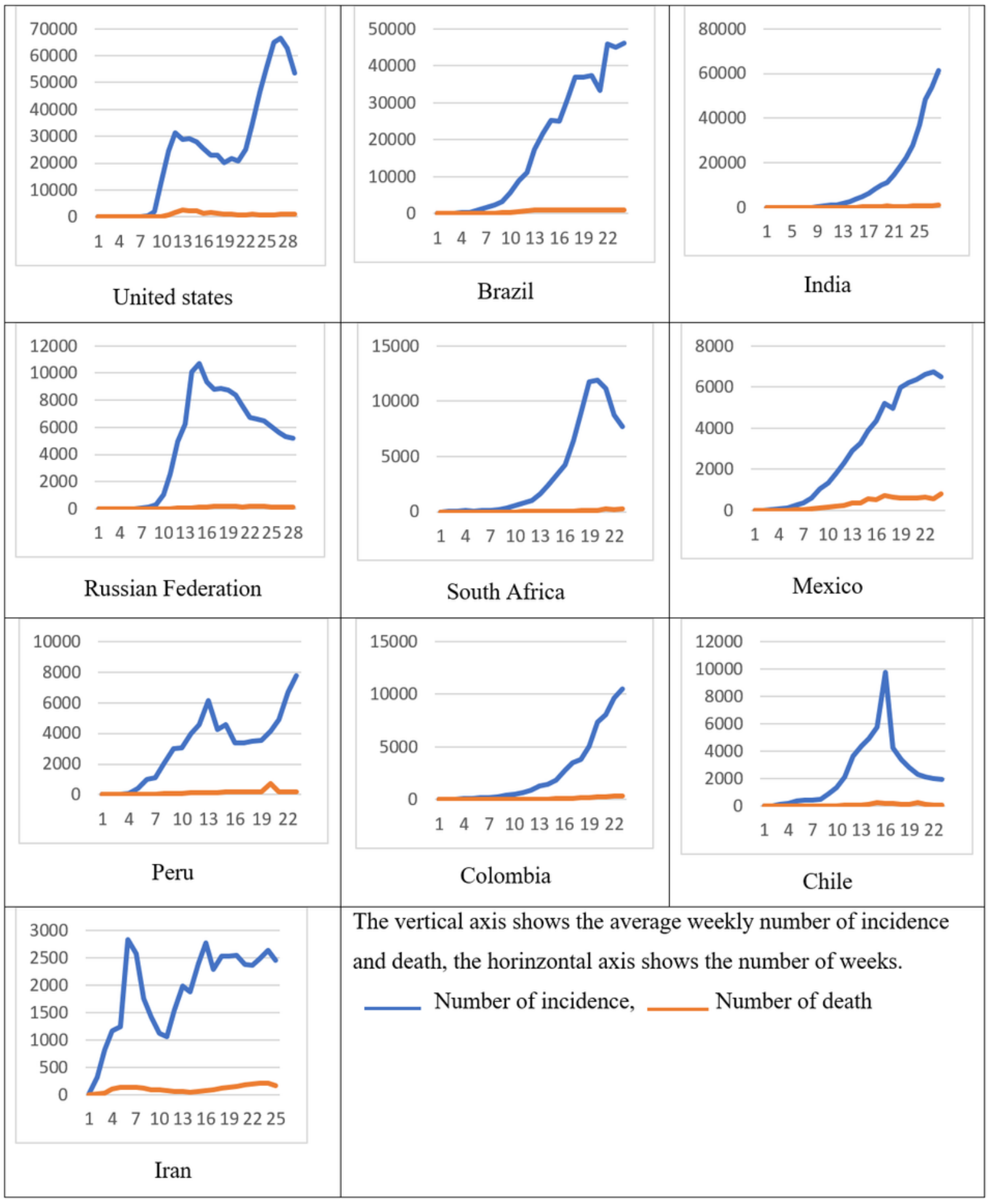

\section{Figure 1}

The trend in the number of incidence and death due to COVID-19 until 10 August, 2020 in selected countries 